\title{
Entropy analysis of integer and fractional dynamical systems
}

\author{
J.A. Tenreiro Machado
}

\begin{abstract}
This paper investigates the adoption of entropy for analyzing the dynamics of a multiple independent particles system. Several entropy definitions and types of particle dynamics with integer and fractional behavior are studied. The results reveal the adequacy of the entropy concept in the analysis of complex dynamical systems.
\end{abstract}

Keywords Fractional calculus · Entropy ·

Dynamics $\cdot$ Complex systems

\section{Introduction}

In the last two decades we witnessed an increasing interest in the generalization of the classical concepts of differential calculus and of entropy. The notion of 'Fractional calculus' (FC) stems from Leibniz [1-5], but only recently relevant applications emerged in the areas of physics and engineering [6-18]. The concept of entropy was introduced in the field of thermodynamics by Clausius (1862) and Boltzmann (1896) and was later applied by Shannon (1948) and Jaynes
(1957) to information theory [19-22]. However, recently more general entropy measures have being proposed, allowing the relaxation of the additivity axiom for application in several types of complex systems [23-30].

The novel ideas are presently under large development and open up ambitious perspectives. Bearing these facts in mind, the present study combines both concepts in the analysis of dynamical systems and is organized as follows. Section 2 introduces a brief description of the fractional calculus and the entropy. Section 3 formulates the conditions underlying the integer and fractional order dynamical system and develops their analysis through several entropy measures. Finally, Sect. 4 outlines the main conclusions.

\section{Fundamental concepts}

This section presents the main mathematical tools adopted in this study, namely the fractional calculus and the entropy.

\subsection{Fractional calculus}

Fractional calculus (FC) is a generalization of the ordinary integer differentiation and integration to an arbitrary order. The subject was initiated in 1695 by Leibniz that sent a letter to L'Hospital with the question: "Can the meaning of derivatives with integer order be generalized to derivatives with non-integer orders?". 
FC was an ongoing topic in the last three centuries and many mathematicians, such as Liouville, Riemann and Weyl, contributed to its development.

There are several definitions of fractional derivatives, three of the most important being the RiemannLiouville, the Grunwald-Letnikov, and the Caputo ones, given by:

$$
\begin{gathered}
{ }_{a} D_{t}^{\alpha} f(t)=\frac{1}{\Gamma(n-\alpha)} \frac{d^{n}}{d t^{n}} \int_{a}^{t} \frac{f(\tau)}{(t-\tau)^{\alpha-n+1}} d \tau \\
n-1<\alpha<n \\
{ }_{a} D_{t}^{\alpha} f(t)=\lim _{h \rightarrow 0} \frac{1}{h^{\alpha}} \sum_{k=0}^{\left[\frac{t-a}{h}\right]}(-1)^{k}\left(\begin{array}{l}
\alpha \\
k
\end{array}\right) f(t-k h) \\
{ }_{a} D_{t}^{\alpha} f(t)=\frac{1}{\Gamma(\alpha-n)} \int_{a}^{t} \frac{f^{(n)}(\tau)}{(t-\tau)^{\alpha-n+1}} d \tau \\
n-1<\alpha<n
\end{gathered}
$$

where $\Gamma()$ is Euler's gamma function, $[x]$ means the integer part of $x$, and $h$ is the step time increment.

It is also possible to generalize several results based on transforms, yielding expressions such as the Laplace expression:

$$
\begin{aligned}
& L\left\{{ }_{0} D_{t}^{\alpha} f(t)\right\} \\
& \quad=s^{\alpha} L\{f(t)\}-\sum_{k=0}^{n-1} s^{k}{ }_{0} D_{t}^{\alpha-k-1} f 0^{+}
\end{aligned}
$$

where $s$ and $L$ represent the Laplace variable and operator, respectively.

These definitions demonstrate that fractional derivatives capture the history of the variable, or, in other words, have memory, contrary to integer derivatives, that are local operators.

The Mittag-Leffler function $E_{\alpha}(x)$ arises in the solution of fractional integral equations and interpolates between a purely exponential law and a power-like behavior for phenomena governed by ordinary equations and their fractional counterparts. The function $E_{\alpha}(x)$ is defined by:

$E_{\alpha}(x)=\sum_{k=0}^{\infty} \frac{x^{k}}{\Gamma(\alpha k+1)}$

In particular, when $\alpha=1$ we have $E_{1}(x)=e^{x}$. An important characteristic of the Mittag-Leffler function is its asymptotic behavior. In the case of the argument $x \leq 0$, the Mittag-Leffler function decreases monotonically and, for large values of $x$, we can write:

$E_{\alpha}(-x) \approx \frac{1}{\Gamma(1-\alpha)} \frac{1}{x}, \quad \alpha \neq 1$

The Laplace transform yields:

$\left.L\left\{E_{\alpha} \pm a t^{\alpha}\right)\right\}=\frac{s^{\alpha-1}}{s^{\alpha} \mp a}$

Therefore, we see a natural extension of the Laplace transform pairs for the exponential function in terms of integer powers of $s$ to the Mittag-Leffler function in terms of fractional powers of the transform parameter $s$.

The Grunwald-Letnikov formulation inspires the numerical calculation of the fractional derivative based on the approximation of the time increment $h$ through the sampling period $T$ and the series truncation at the $r$ th term. This method is often denoted as Power Series Expansion (PSE) yielding the equation in the $z$ domain:

$\left.Z\left\{D^{\alpha} x(t)\right\} \approx \frac{1}{T^{\alpha}} \sum_{k=0}^{r} \frac{(-1)^{k} \Gamma(\alpha+1)}{k ! \Gamma(\alpha-k+1)} z^{-k}\right] X(z)$

where $X(z)=Z\{x(t)\}$ and $z$ and $Z$ represent the $z$ transform variable and operator, respectively.

In fact, expression (2) represents the Euler (or first backward difference) approximation in the $s \rightarrow z$ conversion scheme, the Tustin approximation being another possibility. The Euler and Tustin rational expressions, $G_{0}\left(z^{-1}\right)=\frac{1}{T}\left(1-z^{-1}\right)$ and $G_{1}\left(z^{-1}\right)=\frac{2}{T} \frac{1-z^{-1}}{1+z^{-1}}$ respectively, are often called generating approximants of zero and first order, respectively. Therefore, the generalization of these conversion methods leads to the non-integer order $\alpha$ results:

$$
\begin{aligned}
& s^{\alpha} \approx \frac{1}{T} 1-z^{-1}=G_{0}^{\alpha} z^{-1} \\
& s^{\alpha} \approx\left(\frac{2}{T} \frac{1-z^{-1}}{1+z^{-1}}\right)^{\alpha}=G_{1}^{\alpha} z^{-1}
\end{aligned}
$$

We can obtain a family of fractional differentiators generated by $G_{0}^{\alpha}\left(z^{-1}\right)$ and $G_{1}^{\alpha}\left(z^{-1}\right)$ weighted by the factors $p$ and $1-p$, yielding:

$s^{\alpha} \approx p G_{0}^{\alpha} z^{-1}+(1-p) G_{1}^{\alpha} z^{-1}$ 
For example, the Al-Alaoui operator corresponds to an interpolation of the Euler and Tustin rules with weighting factor $p=3 / 4$ [31].

In order to get a rational expression, the final approximation corresponds to a PSE or a rational fraction expansion. This approach is often denoted by Continued Fraction Expansion (CFE) of order $k \in \aleph$, based on a Padé expansion in the neighborhood of $z^{-1}=0$, yielding:

$G_{k} z^{-1}=\frac{\sum_{i=0}^{k} a_{i} z^{-i}}{\sum_{i=0}^{k} b_{i} z^{-i}}, \quad a_{i}, b_{i} \in \Re$

Since one parameter is linearly dependent, usually it is established that $b_{0}=1$.

\subsection{Entropy}

Khinchin formulated four axioms for a 'classical' (i.e., yielding an ordinary Boltzmann-Gibbs statistical mechanics) measure $H$, namely:

Axiom 1: $\quad H=H\left(p_{1}, \ldots, p_{W}\right)$

Axiom 2: $\quad H W^{-1}, \ldots, W^{-1} \geq H\left(p_{1}, \ldots, p_{W}\right)$

Axiom 3: $\quad H\left(p_{1}, \ldots, p_{W}\right)=H\left(p_{1}, \ldots, p_{W}, 0\right)$

Axiom 4: $\quad H\left(\left\{p_{i j}^{A, B}\right\}\right)$

$$
=H\left(\left\{p_{i}^{A}\right\}\right)+\sum_{i} p_{i}^{A} H\left(\left\{p_{i}^{B}(i \mid j)\right\}\right)
$$

where $W$ represents the number of possible events and $p_{i}$ is the probability that event $i$ occurs, so that $\sum_{i=1}^{W} p_{i}=1$.

Axiom 1 means that $H$ only depends on the probabilities $p_{i}, i=1, \ldots, W$. Axiom 2 states that $H$ takes a maximum for the uniform probability distribution (i.e., all probabilities are equal to $p_{i}=W^{-1}$ ). Axiom 3 says that $H$ does not change if the sample set is enlarged by another event with zero probability. Axiom 4 postulates that given two systems $A$ and $B$, not necessarily independent, $H$ should be independent of the way information is collected. When systems $A$ and $B$ are independent, $p_{i j}^{A, B}=p_{i}^{A} p_{j}^{B}$ results and axiom 4 reduces to the rule of additivity:

$$
H\left(\left\{p_{i j}^{A, B}\right\}\right)=H\left(\left\{p_{i}^{A}\right\}\right)+H\left(\left\{p_{j}^{B}\right\}\right)
$$

This condition is less stringent than axiom (15) and states that the entropy of independent systems should be additive.

The most celebrated entropy is the so-called Shannon entropy $S$ defined by:

$S=-\sum_{i=1}^{W} p_{i} \ln \left(p_{i}\right)$

which satisfies the four Shannon-Khinchin axioms (12)-(14).

The Shannon entropy represents the expected value of the information $-\ln \left(p_{i}\right)$. Therefore, for the uniform probability distribution we have $p_{i}=W^{-1}$ and the Shannon entropy takes its maximum value $S=\ln (W)$, yielding Boltzmann's famous formula, up to a multiplicative factor $k$ denoting the Boltzmann constant. Therefore, in thermodynamic equilibrium, the Shannon entropy can be identified as the 'physical entropy' of the system.

Two of the most studied generalizations of the entropy are the Rényie and Tsallis entropies given by:

$$
\begin{aligned}
S_{q}^{(R)} & =\frac{1}{1-q} \ln \left(\sum_{i=1}^{W} p_{i}^{q}\right), \quad q>0 \\
S_{q}^{(T)} & =\frac{1}{q-1}\left(1-\sum_{i=1}^{W} p_{i}^{q}\right)
\end{aligned}
$$

which reduce to the Shannon entropy when $q \rightarrow 1$.

Recently Ubriaco [32] proposed the fractional entropy:

$S_{q}^{(U)}=\sum_{i=1}^{W}\left(-\ln p_{i}\right)^{q} p_{i}$

which has the same properties as the Shannon entropy except additivity.

Several other measures were proposed, such as the Landsberg-Vedral, Abel, Kaniagakis and SharmaMittal entropies.

\section{Entropy analysis of integer and fractional dynamical systems}

In this section we analyze integer and fractional order dynamical systems through the entropy measure. 
Fig. 1 Evolution of $S(t)$ (thicker trace), $S_{q}^{(R)}(t)$, $S_{q}^{(T)}(t)$ and $S_{q}^{(U)}(t)$ for $q=\{1 / 2,2\}$ for a system composed by $n=10^{4}$ independent particles having dynamics given by expression (21) with $a=1.0$

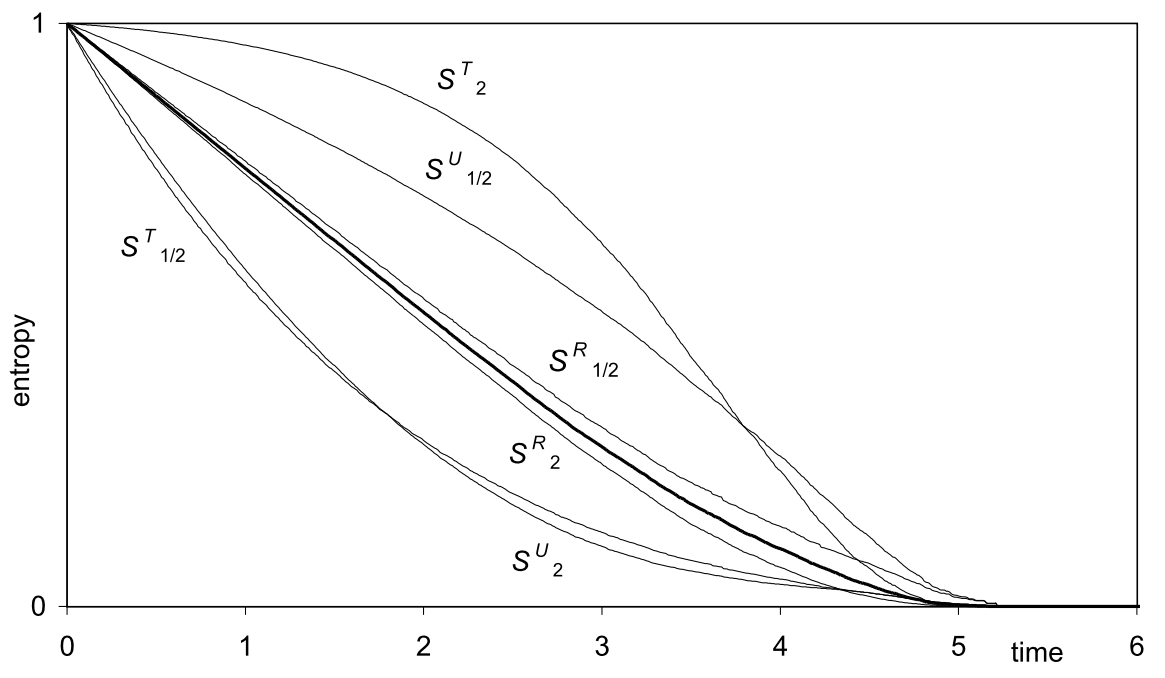

We consider an isolated system consisting of $n$ independent particles, each one having a one-dimensional trajectory evolution $x_{i}(t), i=1, \ldots, n$. There is neither dynamical interaction between particles (i.e., the probability of collisions between the particles is zero) nor impacts with some container or walls. Therefore, the system dynamical state is characterized by the phase space $\{X, \dot{X}\} \equiv\left\{\left(x_{i}, \dot{x}_{i}\right)\right.$, $i=1, \ldots, n\}$ and each particle has a specific dynamics.

In this paper the particle dynamics is considered to be one of the following integer and fractional order test cases:

$$
\begin{aligned}
& x_{i}^{(1)}+a x_{i}=0, \quad i=1, \ldots, n \\
& x_{i}^{(2)}+a x_{i}^{(1)}+b x_{i}=0, \quad i=1, \ldots, n \\
& x_{i}^{(\alpha)}+a x_{i}=0, \quad 0<\alpha<1, i=1, \ldots, n \\
& x_{i}^{(1+\alpha)}+a x_{i}^{(1)}+b x_{i}^{(\alpha)}+c x_{i}=0, \\
& \quad 0<\alpha<1, i=1, \ldots, n
\end{aligned}
$$

It is adopted that $n=10^{4}$ and the initial conditions are generated by a normalized Gaussian distribution $N(0,1)$ with zero average and unit standard deviation. For the cases (23)-(24) the fractional derivatives are evaluated numerically through the PSE method (8), with $r=10$, and the initialization is completed numerically by a preliminary simulation of $r$ iterations. Moreover, in all cases is adopted an integration step time of $d t=10^{-2} \mathrm{sec}$.
The system global state is measured through the entropies $S, S_{q}^{(R)}, S_{q}^{(T)}, S_{q}^{(U)}$ and, for the Rényie, Tsallis and Ubriaco entropy measures, the cases $q=\{1 / 2,2\}$ are evaluated. The theoretical probabilities are approximated through the relative frequencies of occurrence. For this purpose, in each time step $d t$, is constructed a two-dimensional histogram characterizing the phase space $\{X, \dot{X}\}$ with $N_{1} \times N_{2}=10^{2} \times 10^{2}$ bins in the range $N_{1}:-\max \left(x_{i}\right)<x_{i}<\max \left(x_{i}\right)$ and $N_{2}:-\max \left(x_{i}^{(1)}\right)<x_{i}^{(1)}<\max \left(x_{i}^{(1)}\right)$. Therefore, by applying to the phase-space dynamics a time-sliding window with duration $d t$, a sequence of time-stamped values $S(t), S_{q}^{(R)}(t), S_{q}^{(T)}(t), S_{q}^{(U)}(t)$ is obtained, producing a particular entropy curve that depends on the system, the entropy formula and the time. Furthermore, in order to simplify the comparison the different measures are rescaled so that we get an evolution between one and zero.

Figures 1-4 show the seven cases of entropy measuring when applied to systems with particle dynamics described by (21)-(24), respectively. The Shannon entropy $S$ is represented with a thicker trace. As expected we verify that, in all cases, the entropy decreases monotonically and that the $S(t)$ plot is in the 'middle' of the traces corresponding to $S_{q}^{(R)}(t), S_{q}^{(T)}(t)$, $S_{q}^{(U)}(t)$ for $q=\{1 / 2,2\}$. As concerns the global system dynamics we observe that the first order equation (21) leads to a single phase evolution, while the second order equation (22) leads to a more 'sophisticated' behavior. The fractional dynamics equations (23) and (24) somehow interpolate the integer order cases. 
Fig. 2 Evolution of $S(t)$ (thicker trace), $S_{q}^{(R)}(t)$, $S_{q}^{(T)}(t)$ and $S_{q}^{(U)}(t)$ for $q=\{1 / 2,2\}$ for a system composed by $n=10^{4}$ independent particles having dynamics given by expression (22) with $a=3.0, b=2.0$

Fig. 3 Evolution of $S(t)$ (thicker trace), $S_{q}^{(R)}(t)$, $S_{q}^{(T)}(t)$ and $S_{q}^{(U)}(t)$ for $q=\{1 / 2,2\}$ for a system composed by $n=10^{4}$ independent particles having dynamics given by expression (23) with $\alpha=0.5, a=1.0$
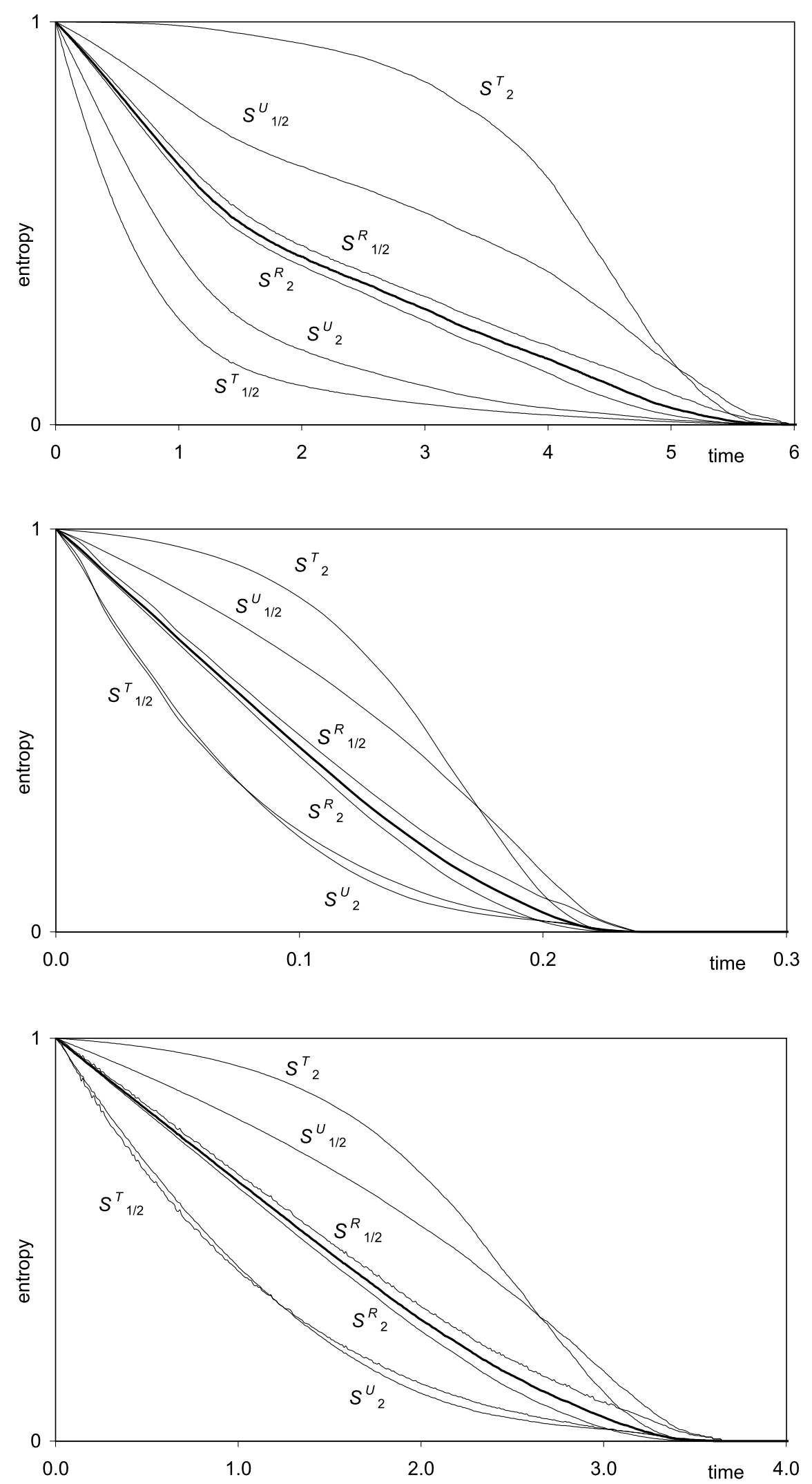

Fig. 4 Evolution of $S(t)$ (thicker trace), $S_{q}^{(R)}(t)$, $S_{q}^{(T)}(t)$ and $S_{q}^{(U)}(t)$ for $q=\{1 / 2,2\}$ for a system composed by $n=10^{4}$ independent particles having dynamics given by expression (24) with $\alpha=0.5, a=1.0, b=2.0$ and $c=2.0$ 
Fig. 5 Evolution of $S(t)$ for a system composed by $n=10^{4}$ independent particles having dynamics given by expression (22) with $(a, b)=\{(2,1),(3,2)$, $(4,3),(5,4)\}$

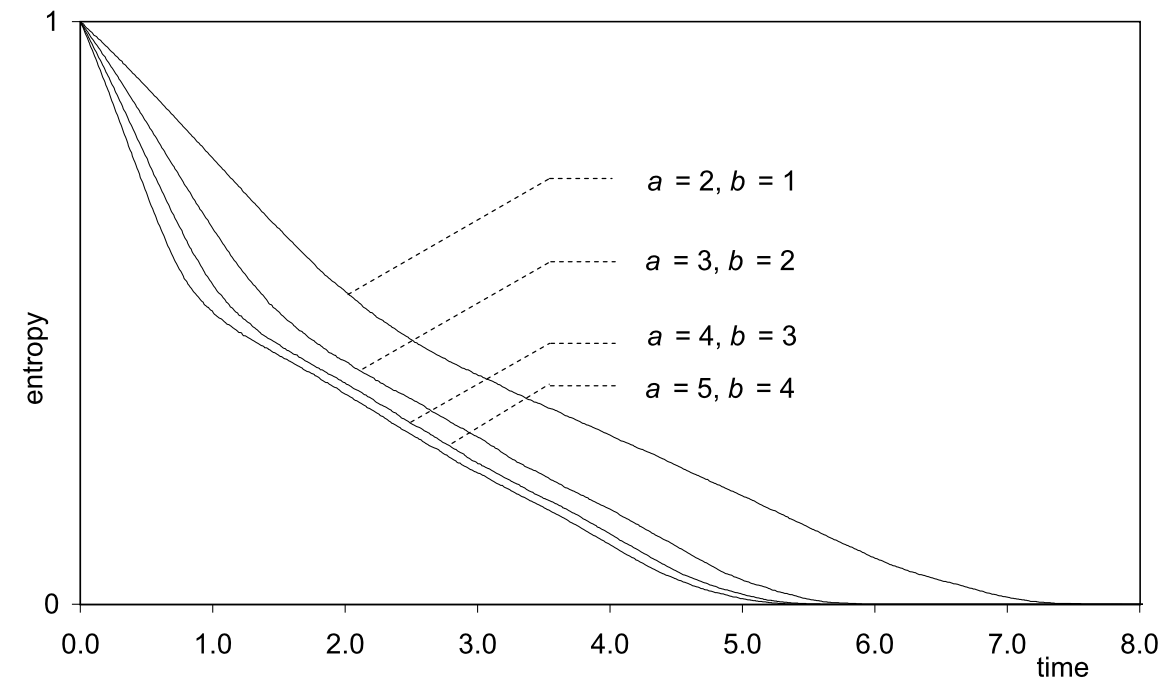

Fig. 6 Evolution of $S(t)$ for a system composed by $n=10^{4}$ independent particles having dynamics given by expression (24) with

$\alpha=\{0.1,0.3,0.5,0.7,0.9\}$,

$a=1.0, b=2.0$ and

$c=2.0$

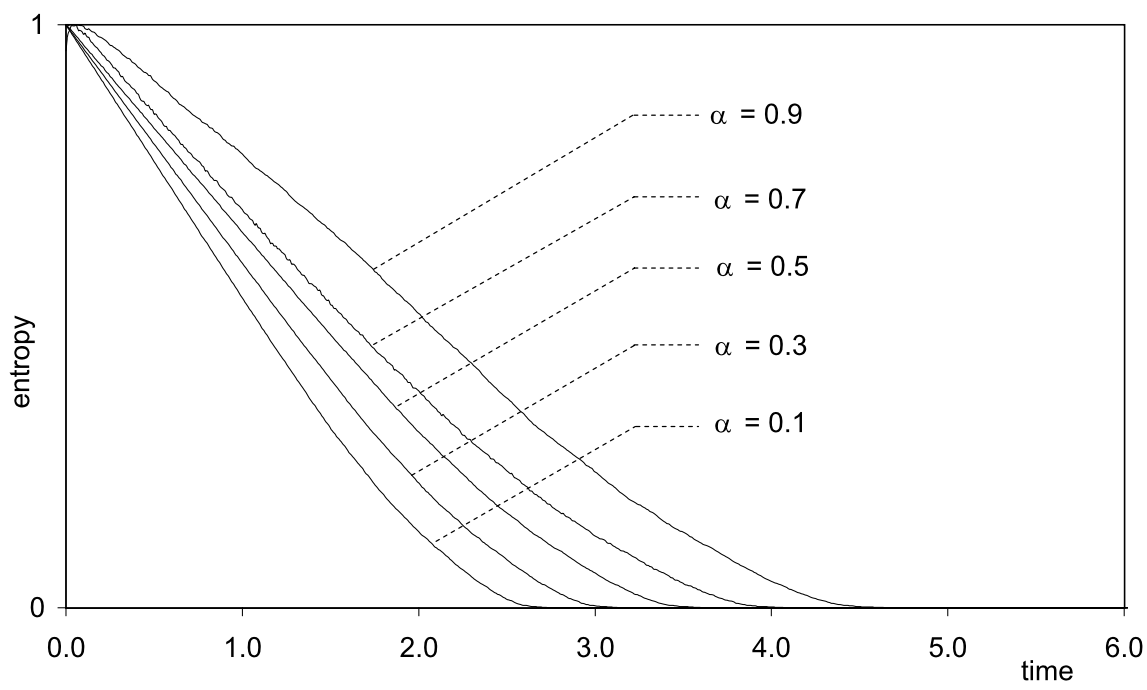

Figures 5-6 show the variation of $S(t), S_{q}^{(R)}(t)$, $S_{q}^{(T)}(t), S_{q}^{(U)}(t)$ for different eigenvalues in (22) and distinct fractional orders $\alpha$ in (24).

In the simulations the initial state was totally disordered and, therefore, it has maximal entropy normalized to one. For time increasing the systems tend to a final state of static or dynamic equilibrium with entropy zero.

We conclude that the entropy characterizes adequately the dynamical evolution of the multiple particle system. The tested cases did not show any pros or cons for a particular entropy measure. Since the benchmarks were developed for an isolated system, without any type of further dynamical interaction, the behav- ior of more complex systems remains to be investigated.

The time evolution of the entropy plotted in the charts can be approximated easily and with good accuracy by polynomials or rational fractions; nevertheless, under the process of numerical fitting, the dynamic behavior will be hidden. Bearing this idea in mind, an alternative strategy was devised and the asymptotic behavior, that is, the evolution at the beginning or at the end of the time evolution, was approximated numerically by independent functions. In order to avoid the perturbation introduced by the bin counting for the relative frequency calculation when the entropy is close to zero, the final values were dis- 
regarded. For the Shannon entropy the approximations of the initial $S_{\text {initial }}(t)$ and final $S_{\text {final }}(t)$ evolutions yielded the results:

- Fig. 1:

$$
S_{\text {initial }}(t) \approx 1-0.25 t, \quad S_{\text {final }}(t) \sim-0.62 \ln t
$$

- Fig. 2:

$$
S_{\text {initial }}(t) \approx 1-0.36 t, \quad S_{\text {final }}(t) \sim-0.43 \ln t
$$

- Fig. 3:

$$
S_{\text {initial }}(t) \approx 1-5.4 t, \quad S_{\text {final }}(t) \sim-0.62 \ln t
$$

- Fig. 4:

$$
S_{\text {initial }}(t) \approx 1-0.35 t, \quad S_{\text {final }}(t) \sim-0.05 \ln t
$$

- Fig. 5:

$$
\begin{array}{ll}
(a, b)=(2,1), & S_{\text {initial }}(t) \approx 1-0.24 t \\
& S_{\text {final }}(t) \sim-0.40 \ln t \\
(a, b)=(2,1), & S_{\text {initial }}(t) \approx 1-0.31 t \\
& S_{\text {final }}(t) \sim-0.38 \ln t \\
(a, b)=(2,1), & S_{\text {initial }}(t) \approx 1-0.47 t \\
& S_{\text {final }}(t) \sim-0.38 \ln t \\
(a, b)=(2,1), & S_{\text {initial }}(t) \approx 1-0.59 t \\
& S_{\text {final }}(t) \sim-0.40 \ln t
\end{array}
$$

- Fig. 6:

$$
\begin{array}{ll}
\alpha=0.1, & S_{\text {initial }}(t) \approx 1-0.47 t \\
\alpha=0.3, & S_{\text {final }}(t) \sim-0.61 \ln t \\
& S_{\text {initial }}(t) \approx 1-0.41 t \\
& S_{\text {final }}(t) \sim-0.61 \ln t \\
\alpha=0.5, & S_{\text {initial }}(t) \approx 1-0.35 t \\
& S_{\text {final }}(t) \sim-0.61 \ln t \\
\alpha=0.7, & S_{\text {initial }}(t) \approx 1-0.32 t \\
& S_{\text {final }}(t) \sim-0.59 \ln t \\
\alpha=0.9, & S_{\text {initial }}(t) \approx 1-0.25 t \\
& S_{\text {final }}(t) \sim-0.63 \ln t
\end{array}
$$

We verify that while the initial transient has a fast linear time variation, the final dynamics is much slower, revealing a logarithmic time dependence.

While the focus of the present study was mainly the comparison of different entropy formulations for integer and fractional dynamical systems, another important aspect deserves future attention. In fact, it was assumed that the effect of interaction terms between the subsystems is negligible in comparison with the effects of their own equations of motion. Nevertheless, in complex systems interaction effects modify the dynamics [33] and the resulting decrease of entropy remains to be investigated, which will be pursued in future research.

\section{Conclusions}

This paper reviewed two important mathematical tools, namely the fractional calculus and the entropy. These concepts allow a fruitful interplay in the analysis of system dynamics. Nevertheless, the synergies of applying both tools has been, somehow, neglected in engineering and applied sciences. The paper analyzed multi-particle systems with integer and fractional order behavior and demonstrated that the concepts are simple and straightforward to apply. In this line of thought, future research will address the analysis of more complex systems.

Acknowledgement The author would like to thank the anonymous reviewers for their insightful comments, which helped to improve this paper.

\section{References}

1. Oldham, K.B., Spanier, J.: The Fractional Calculus: Theory and Application of Differentiation and Integration to Arbitrary Order. Academic Press, New York (1974)

2. Samko, S.G., Kilbas, A.A., Marichev, O.I.: Fractional Integrals and Derivatives: Theory and Applications. Gordon \& Breach, New York (1993)

3. Miller, K.S., Ross, B.: An Introduction to the Fractional Calculus and Fractional Differential Equations. Wiley, New York (1993)

4. Podlubny, I.: Fractional Differential Equations. An Introduction to Fractional Derivatives, Fractional Differential Equations, to Methods of Their Solution. Mathematics in Science and Engineering, vol. 198. Academic Press, New York (1998)

5. Kilbas, A.A., Srivastava, H.M., Trujillo, J.J.: Theory and Applications of Fractional Differential Equations. NorthHolland Mathematics Studies, vol. 204. Elsevier, Amsterdam (2006)

6. Oustaloup, A.: La Commande CRONE: Commande Robuste D'ordre non Entier. Hermes, Oslo (1991)

7. Anastasio, T.J.: The fractional-order dynamics of brainstem vestibulo-oculomotor neurons. Biol. Cybern. 72(1), 69-79 (1994) 
8. Mainardi, F.: Fractional relaxation-oscillation and fractional diffusion-wave phenomena. Chaos Solitons Fractals 7, 1461-1477 (1996)

9. Machado, J.T.: Analysis and design of fractional-order digital control systems. J. Syst. Anal., Model. Simul. 27, 107$122(1997)$

10. Nigmatullin, R.: The statistics of the fractional moments: Is there any chance to "read quantitatively" any randomness? Signal Process. 86(10), 2529-2547 (2006)

11. Tarasov, V.E., Zaslavsky, G.M.: Fractional dynamics of systems with long-range interaction. Commun. Nonlinear Sci. Numer. Simul. 11(8), 885-898 (2006)

12. Baleanu, D.: About fractional quantization and fractional variational principles. Commun. Nonlinear Sci. Numer. Simul. 14(6), 2520-2523 (2009)

13. Podlubny, I.: Fractional-order systems and $\mathrm{PI}^{\lambda} \mathrm{D}^{\mu}$ controllers. IEEE Trans. Autom. Control 44(1), 208-213 (1999)

14. Tenreiro Machado, J.: Discrete-time fractional-order controllers. J. Fract. Calc. Appl. Anal. 4, 47-66 (2001)

15. Chen, Y.Q., Moore, K.L.: Discretization schemes for fractional-order differentiators and integrators. IEEE Trans. Circuits Syst. I, Fundam. Theory Appl. 49(3), 363-367 (2002)

16. Tseng, C.C.: Design of fractional order digital fir differentiators. IEEE Signal Process. Lett. 8(3), 77-79 (2001)

17. Vinagre, B.M., Chen, Y.Q., Petras, I.: Two direct Tustin discretization methods for fractional-order differentiator/integrator. J. Franklin Inst. 340(5), 349-362 (2003)

18. Tenreiro Machado, J., Galhano, A.M.S.: Statistical fractional dynamics. ASME J. Comput. Nonlinear Dyn. 3(2), 021201 (2008)

19. Shannon, C.E.: A mathematical theory of communication. Bell Syst. Tech. J. 27, 379-423 (1948)

20. Shannon, C.E.: A mathematical theory of communication. Bell Syst. Tech. J. 27, 623-656 (1948)
21. Jaynes, E.T.: Information theory and statistical mechanics. Phys. Rev. 106, 620 (1957)

22. Khinchin, A.I.: Mathematical Foundations of Information Theory. Dover, New York (1957)

23. Plastino, A., Plastino, A.R.: Tsallis entropy and Jaynes' information theory formalism. Braz. J. Phys. 29(1), 50-60 (1999)

24. Li, X., Essex, C., Davison, M., Hoffmann, K.H., Schulzky, C.: Fractional diffusion, irreversibility and entropy. J. NonEquilib. Thermodyn. 28(3), 279-291 (2003)

25. Haubold, H.J., Mathai, A.M., Saxena, R.K.: BoltzmannGibbs entropy versus Tsallis entropy: recent contributions to resolving the argument of Einstein concerning "Neither Herr Boltzmann nor Herr Planck has given a definition of W'? Astrophys. Space Sci. 290(3-4), 241-245 (2004)

26. Mathai, A.M., Haubold, H.J.: Pathway model, superstatistics, Tsallis statistics, and a generalized measure of entropy. Phys. A, Stat. Mech. Appl. 375(1), 110-122 (2007)

27. Carter, T.: An introduction to information theory and entropy. Complex Systems Summer School, Santa Fe (June 2007)

28. Rathie, P., Da Silva, S.: Shannon, Levy, and Tsallis: a note. Appl. Math. Sci. 2(28), 1359-1363 (2008)

29. Beck, C.: Generalised information and entropy measures in physics. Contemp. Phys. 50(4), 495-510 (2009)

30. Gray, R.M.: Entropy and Information Theory. Springer, Berlin (2009)

31. Al-Alaoui, M.A.: Novel digital integrator and differentiator. Electron. Lett. 29(4), 376-378 (1993)

32. Ubriaco, M.R.: Entropies based on fractional calculus. Phys. Lett. A 373(30), 2516-2519 (2009)

33. Tenreiro Machado, J., Galhano, A.M.: Statistical fractional dynamics. ASME J. Comput. Nonlinear Dyn. 3(2), 021201 (2008) 